Supporting information for:

\title{
Unveiling of swainsonine biosynthesis via a multi-branched pathway in fungi
}

Feifei Luo, Song Hong, Bo Chen, Ying Yin, Guirong Tang, Fenglin Hu, Huizhan Zhang, Chengshu Wang*

Key Laboratory of Insect Developmental and Evolutionary Biology, CAS Center for Excellence in Molecular Plant Sciences, Institute of Plant Physiology and Ecology, Chinese Academy of Sciences, Shanghai, China.

*Corresponding author: wangcs@sippe.ac.cn, tel. 86-21 54924157 


\section{Table of Contents:}

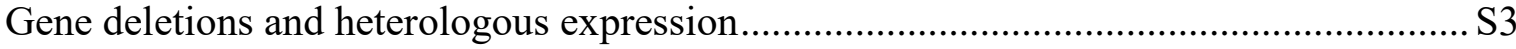

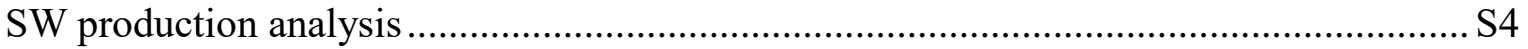

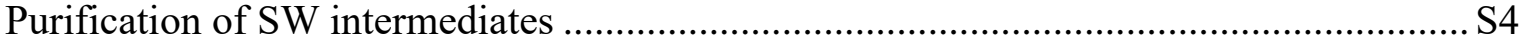

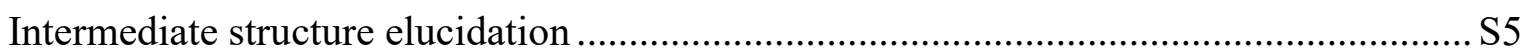

Effect of SW production on fungal interactions with plants ........................................ S6

Figure S1. LC-MS analysis of the EIC signals of the compounds 3, 4a and 4b produced in different fungi.

Figure S2. Functional verification of genes involved in the production of pipecolic acid (PA) and swainsonine (SW). S8

Figure S3. Sequence alignments of the conserved fungal and bacterial KR and K domains. $\mathrm{S} 10$

Figure S4. The effects of swnN and swnR on the production of different intermediates. S12 Figure S5. Schematic pathways for the conversion of compounds 3 and $4 \mathrm{a} / \mathrm{b}$ through the formation and reduction of iminium ions $\mathrm{S} 13$

Figure S6. LC-MS analysis of SW production during 5b and 5c feeding assays. S14

Figure S7. Examination of SwnT to the intracellular and extracellular accumulation of

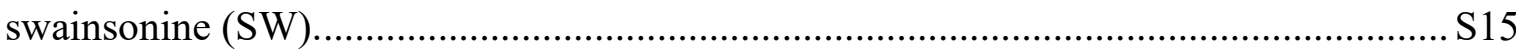

Figure S8. Plant root colonization and insect assays................................................... 16 Figure S9. Gene deletion, overexpression and verification. (A) A schematic map for the

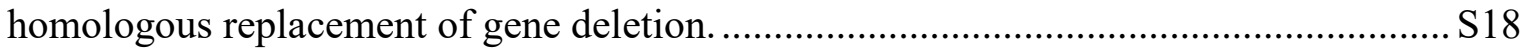
Table S1. NMR data collected for the compound 3 formate in $\mathrm{CDCl}_{3}$ and $4 \mathrm{a}$ formate in $\mathrm{CD}_{3} \mathrm{OD}$. S19

Table S2. NMR data collected for the compounds $5 b$ and $5 c$ formates in $\mathrm{CD}_{3} \mathrm{OD}$......... S20

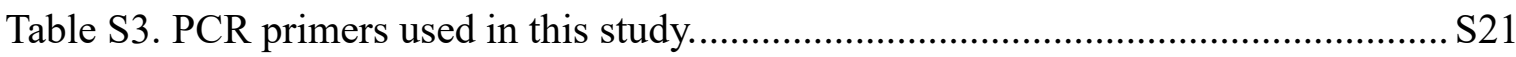

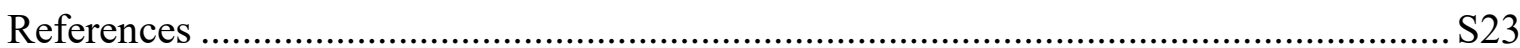




\section{Gene deletions and heterologous expression}

To determine gene functions, the genes encoded in SW BGC and the putative Lys cyclodeaminase genes (MAA_02105 and MAA_10225) located outside the cluster were individually deleted by homologous recombination (Figure S9A) via Agrobacteriummediated transformation of the WT strain ARSEF 2575 of M. robertsii. ${ }^{1}$ Briefly, the 5 '- and 3'-flanking sequences of individual genes were amplified with different primer pairs (Table S3) and Phanta ${ }^{\mathrm{TM}}$ Super-Fidelity DNA Polymerase (Vazyme Biotech) using genomic DNA as a template. The products were digested with the appropriate restriction enzymes and then cloned into the binary vector pDHt-bar (conferring resistance to ammonium glufosinate) treated with the same restriction enzyme to generate knock-out plasmids for fungal transformation. ${ }^{2}$ Drug-resistant colonies were selected and verified by PCR (Figure S9B). To determine the effect of swnA on PA production, full open reading frame (ORF) of swnA was cloned by PCR and made under the control of the constitutive $H s p 70$ gene promoter. ${ }^{3}$ The cassette was then cloned into the binary vector pDHt-bar for transformation of the WT strain of $M$. robertsii. Two independent transformants were selected by reverse transcription-PCR analysis (Figure S9C) and used for further analysis.

The heterologous expression of $s w n K$ gene was conducted in the WT strain of $B$. bassiana. Three fragments of swnK gene were individually amplified by PCR using genomic DNA of $M$. robertsii as a template. The fragments were purified and fused together by PCR using the amplified products as templates and the primer pairs ExF1 and ExF3 (Table S3). The accuracy of $s w n K$ full ORF was verified by sequencing. The gene was placed under of the control of the constitutive $g p d A$ gene promoter, ${ }^{4}$ and the cassette was then cloned into the vector pDHt-bar using the ClonExpress ${ }^{\mathrm{TM}}$ MultiS recombinase (Vazyme Biotech, China). The obtained vector was used to transform the WT strain of $B$. bassiana to generate the mutant BBA::swnK. Both the WT and mutant were grown in $50 \mathrm{ml}$ flasks each containing $20 \mathrm{ml} \mathrm{SDB}$ at $30^{\circ} \mathrm{C}$ and $200 \mathrm{rpm}$ for seven days, and mycelia were collected by filtration and washed twice with distilled water. The samples were then freezedried, homogenized and individually extracted (30 $\mathrm{mg}$ each) with $1 \mathrm{ml}$ of methanol by vortexing for one min and subsequently sonicating for $40 \mathrm{~min}$ for detection of the compounds produced by SwnK.

To further determine the function of $s w n A$, the gene was also expressed in yeast cells. Thus, the cDNA of swnA was amplified and cloned into the pYES2 plasmid to generate the plasmid pYES2-SwnA. In addition, both swnA and swnR were cloned into the bivalent 
expression vector $p Y E S 2-2 p$ to generate the plasmid pYES2-SwnA.R. The plasmids were individually transformed into the strain of $S$. cerevisiae INVSc1 according to the procedure provided by the manufacturer. Yeast strains were inoculated in $10 \mathrm{ml} \mathrm{SD-Ura} \mathrm{medium} \mathrm{(yeast}$ nitrogen base, 6.7 g; glucose, 20 g; Dropout Supplement-Ura 1.29 g; water, 1 liter) and incubated in a rotary shaker at $30{ }^{\circ} \mathrm{C}$ and $220 \mathrm{rpm}$. After the growth reaching an $\mathrm{OD}_{600}$ of 1.0 , the cells were harvested by centrifugation at $5000 \mathrm{rpm}$ for $1 \mathrm{~min}$ and washed three times with sterile distilled water. The cells were transferred to $9 \mathrm{ml} \mathrm{SC-Ura} \mathrm{(yeast} \mathrm{nitrogen} \mathrm{base,}$ 6.7g; Dropout Supplement-Ura, $1.29 \mathrm{~g}$; water, $900 \mathrm{ml}$ ) and cultured for $6 \mathrm{hrs}$ to exhaust cellular glucose. Then, $1 \mathrm{ml}$ of induction solution (D-galactose, $20 \mathrm{~g}$; raffinose, $10 \mathrm{~g}$; water $100 \mathrm{ml}$ ) and the substrate Lys (at a final concentration of $10 \mathrm{mM}$ ) were added and incubated at $30{ }^{\circ} \mathrm{C}$ and $220 \mathrm{rpm}$ for 2 days. The cultures were centrifuged at 15,000 rpm for $5 \mathrm{~min}$, and $1 \mathrm{ml}$ supernatant was dried and re-dissolved in $100 \mu \mathrm{L}$ of $60 \%$ methanol for LC-MS analysis.

\section{SW production analysis}

The harvested mycelium samples were individually extracted (30 mg each) with $1 \mathrm{ml}$ of methanol by vortexing for one min and subsequently sonicating for $40 \mathrm{~min}$. To uncover the function of the putative transporter SwnT, the accumulation of SW in the culture filtrate was also investigated. Both WT and $\Delta s w n T$ were incubated in SDB for one week. After filtration, mycelia were dried and extracted as indicated above, and culture filtrates were concentrated with a rotational vacuum concentrator (Martin Christ) and then re-dissolved ultrasonically in $100 \mu \mathrm{l}$ of methanol. To investigate SW accumulation in fungal spores, conidia were gently scrapped from the two-week old PDA plates with a sterile inoculation loop and collected into a petri dish. Methanol was added (30 $\mathrm{mg}$ in $1 \mathrm{ml}$ methanol) and the samples were individually transferred into $1.5 \mathrm{ml}$ tubes for extraction by vortexing for one min and subsequently sonicating for $40 \mathrm{~min}$. There were three independent replicates for each sample. Mycosed insect cadavers and endophytic plant samples were freeze-dried and homogenized in liquid nitrogen. The samples (30 mg each) were then extracted with $1 \mathrm{ml}$ of methanol. All samples were kept at $4{ }^{\circ} \mathrm{C}$ for $12 \mathrm{~h}$ and then centrifuged at $15,000 \mathrm{rpm}$ for $5 \mathrm{~min}$.

\section{Purification of SW intermediates}

The eluent was individually collected using HILIC column when the following characteristic ions were detected with $\mathrm{m} / \mathrm{z}$ values at 140.10 [3; retention time (RT), $7.5 \mathrm{~min}$ ]; 142.12 (4a; RT $8.5 \mathrm{~min}) ; \mathrm{m} / \mathrm{z} 142.12$ (4b; RT $12 \mathrm{~min}$ ) and 158.11 (mixture 5; RT $9.5 \mathrm{~min}$ ). 
Subsequently, the collected fractions was further subject to LC-MS purification with $0.1 \%$ formic acid in water (A) and $0.1 \%$ formic acid in acetonitrile (B) as the mobile phases: the fraction ion with a $\mathrm{m} / \mathrm{z}$ value at 140.10 (3) eluted at a flow rate of $3.5 \mathrm{ml} \mathrm{min}{ }^{-1}$ with isocratic

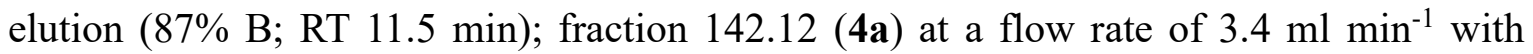
isocratic elution $(72 \% \mathrm{~B}$; RT $7.3 \mathrm{~min})$; fraction $142.12(\mathbf{4 b})$ at a flow rate of $3.3 \mathrm{ml} \mathrm{min}^{-1}$ with isocratic elution (74\% B; RT $10 \mathrm{~min}$ ); and fraction 158.11 (mixture 5) at a flow rate of $3.4 \mathrm{ml} \mathrm{min}-1$ with isocratic elution ( $87 \% \mathrm{~B}$; RT $12 \mathrm{~min}$ ). After this step, the contamination of polar compounds could be still observed in each collection. In particular, a trial NMR analysis indicated that the fraction 158.11 was a mixture. Finally, each fraction was purified using a reversed-phase HPLC column (Atlantis Prep T3; $5 \mu \mathrm{m}, 10 \mathrm{~mm} \times 150 \mathrm{~mm}$ ). The water (A) and methanol (B) containing $1 \mathrm{mM}$ ammonium formate was used as the mobile phase with isocratic elution using $3 \% \mathrm{~B}$ at a flow rate of $2.3 \mathrm{ml} \mathrm{min}{ }^{-1}$. Compound 3 was eluted at RT 4.0 min, and compounds $4 \mathbf{a}$ and $\mathbf{4 b}$ were both eluted at RT $6.5 \mathrm{~min}$. Unexpectedly, fraction 158.11 was eluted into three peaks at RT 6.1, 8.2 and $10.1 \mathrm{~min}$, i.e., for $\mathbf{5 a}, \mathbf{5 b}$ and $\mathbf{5 c}$, respectively. After each step of purification, the obtained fraction were concentrated at $30{ }^{\circ} \mathrm{C}$ using a rotary evaporator or centrifugal concentrator. Prior to NMR analysis, each sample was checked for purity by LC-MS using the CNW Athena C18 analytic column, we found that $\mathbf{4 b}$ was again eluted at the same RT as $\mathbf{4 a}$, and $\mathbf{5 a}$ was eluted at the same RT as $\mathbf{5 b}$. Considering that $4 \mathbf{a}$ and $\mathbf{4 b}$, and $\mathbf{5 a}$ and $\mathbf{5 b}$ have the same $\mathrm{m} / \mathrm{z}$ values with each other, they were pooled together respectively for NMR analysis.

\section{Intermediate structure elucidation}

All the intermediates of SW were purified as formate salts for structure determination. Standard pulse sequences were used for NMR experiments using a Bruker Avance III-500 system. Compound $\mathbf{3}$ formate data were collected in the solvent $\mathrm{CDCl}_{3}$, and $\mathbf{4 a}, \mathbf{5 b}$ and $\mathbf{5 c}$ formats were dissolved in $\mathrm{CD}_{3} \mathrm{OD}$ for data collection. Chemical shifts were referenced to the solvent signal at 7.26 and $77.2 \mathrm{ppm}$ for $\mathrm{CDCl}_{3}$, and 3.31 and $49.0 \mathrm{ppm}$ for $\mathrm{CD}_{3} \mathrm{OD}$. All spectra were processed with the program MestRe Nova (ver. 9.0.1; Metrelab Research, Santiago de Compostella). ${ }^{5}$ To accurately predict the molecular geometries, the reliable quantum chemical calculations were performed using a Gaussian 09 program package. ${ }^{6}$ The NMR isotropic shielding constants were calculated using a gauge independent atomic orbital approach at the B3LYP/6-311+G $(2 d, p)$ level. Solvent effects were taken into 
accounts by using the polarizable continuum model. The ${ }^{1} \mathrm{H}$ chemical shifts were corrected by using the linear regression scaling factors. ${ }^{7}$

\section{Effect of SW production on fungal interactions with plants}

Plant seeds were surface sterilized in $75 \%$ ethanol for $2 \mathrm{~min}$ followed by rinsing in $30 \%$ hydrogen peroxide for $2 \mathrm{~min}$ and then rinsed three times with sterile distilled water. The

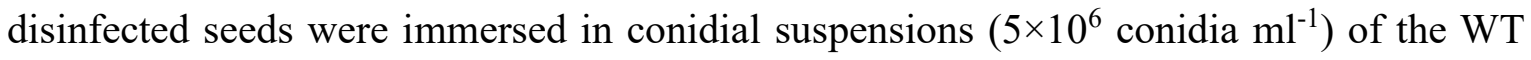
and $\Delta s w n K$ or in sterile distilled water (control treatment) for $16 \mathrm{~h}$. Inoculated and control seeds were placed on wet, sterile filter papers and kept at $25{ }^{\circ} \mathrm{C}$ for germination (2-3 days). Germinated seedlings were transferred to tissue culture bottles and maintained in an artificial climate chamber at $25^{\circ} \mathrm{C}$ and a photoperiod of $16: 8 \mathrm{~h} \mathrm{light/dark}$ for two weeks. Endophytic fungal cells in plant roots or stems were detected by staining with wheat germ agglutinin (WGA-AF488; ThermoFisher). ${ }^{8}$ Alternatively, the inoculated seedlings were planted in disinfected pots filled with sterilized soil (mixture of vermiculite and peat at a ratio of 1:1) and maintained in the greenhouse at $28{ }^{\circ} \mathrm{C}$ for up to 30 days. After plantation, the plants were harvested and roots cleaned for methanol extraction and LC-MS detection of SW accumulation as indicated above. Each plant had at least 10 pots (two plants each pot). The plants collected from independent pots were used as replicates and at least five independent samples were analyzed for each plant. 
A
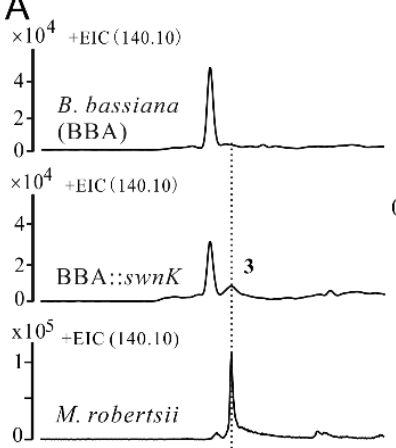

$\mathrm{x} 10^{5}+\mathrm{EIC}(140.10)$

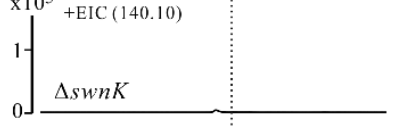

$\times 10^{5}+\operatorname{EIC}(140.10)$
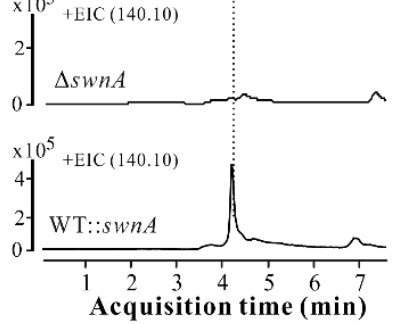
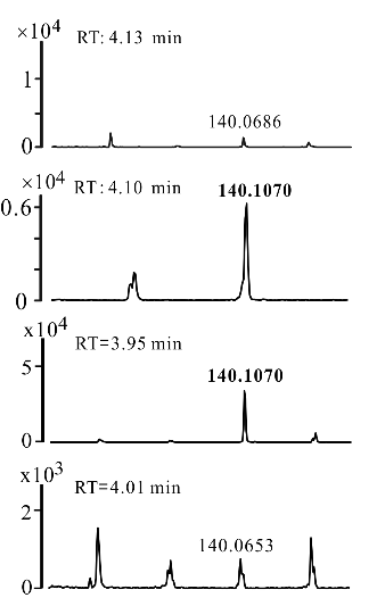

$\mathrm{x} 10^{4} \mathrm{RT}=3.95 \mathrm{~min}$
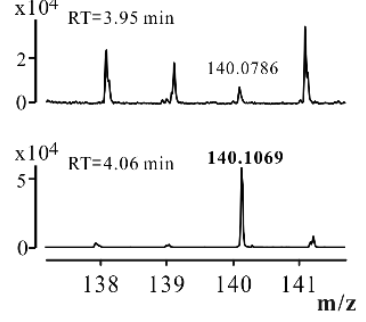

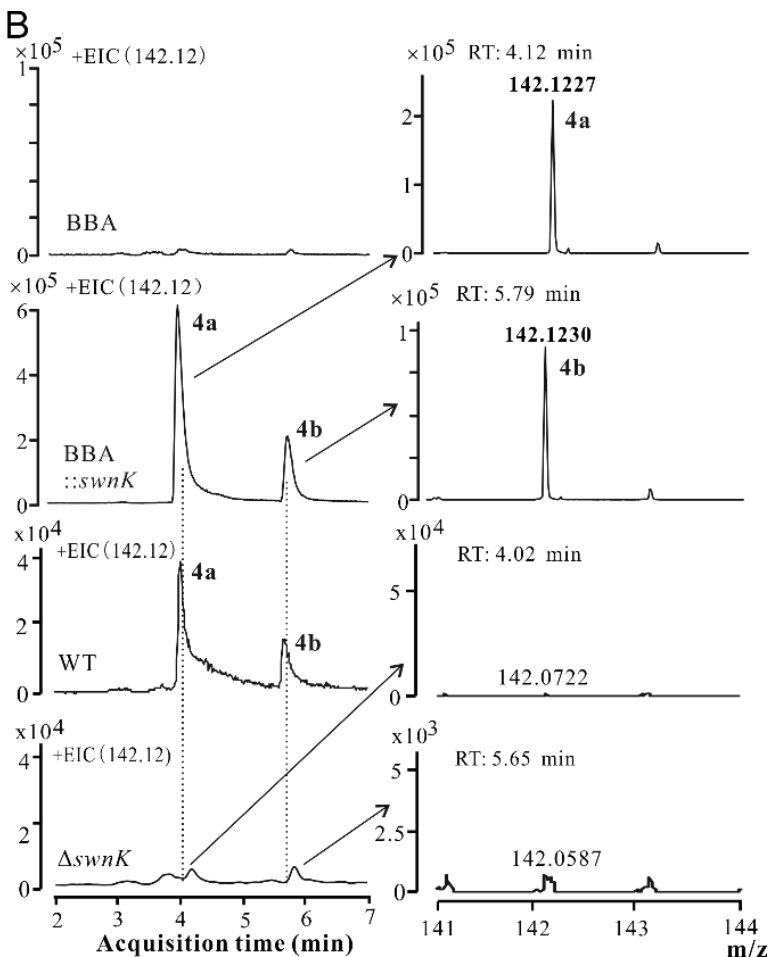

Figure S1. LC-MS analysis of the EIC signals of the compounds 3, $4 \mathrm{a}$ and $4 \mathrm{~b}$ produced in different fungi. (A) LC-MS analysis of compound 3 production in the wild type (WT) and mutants of B. bassiana (BBA) and M. robertsii (labeled at the bottom left of each panel). (B) LC-MS analysis of the compounds $\mathbf{4 a}$ and $\mathbf{4 b}$ produced by BBA::swnK. Left panels show the detection or non-detection of EIC peaks; right panels show the mass spectra of the corresponding compounds ( $\mathrm{m} / \mathrm{z}$ highlighted in bold). 
A $\times 10^{\circ}$
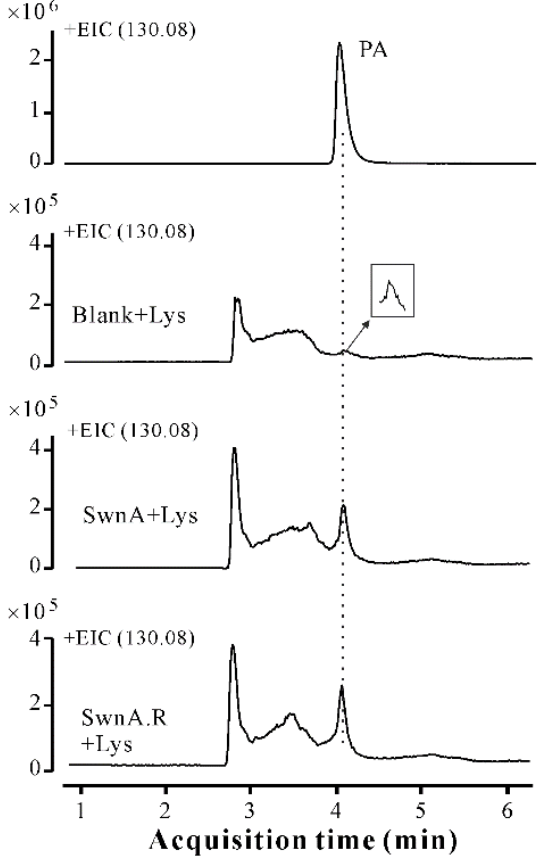

B
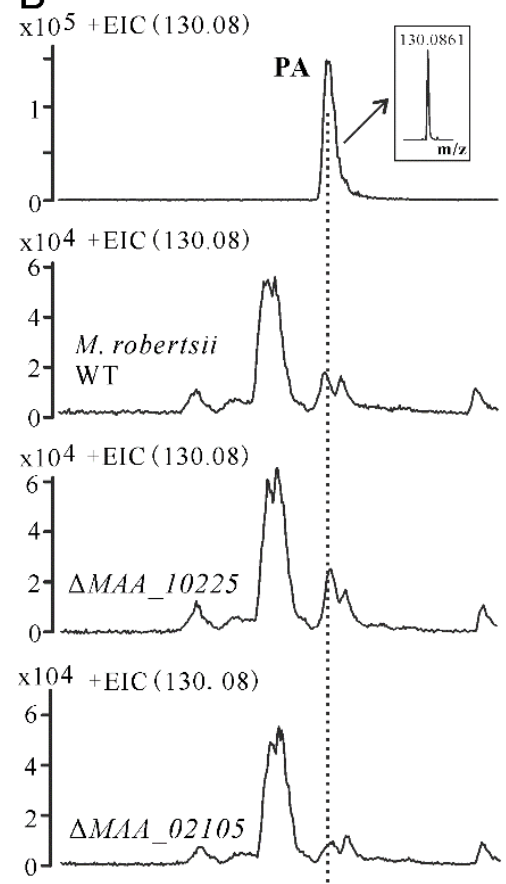

$\mathrm{x} 10^{4}+\mathrm{EIC}(130.08)$

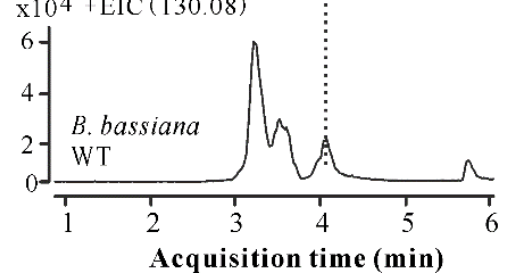

C

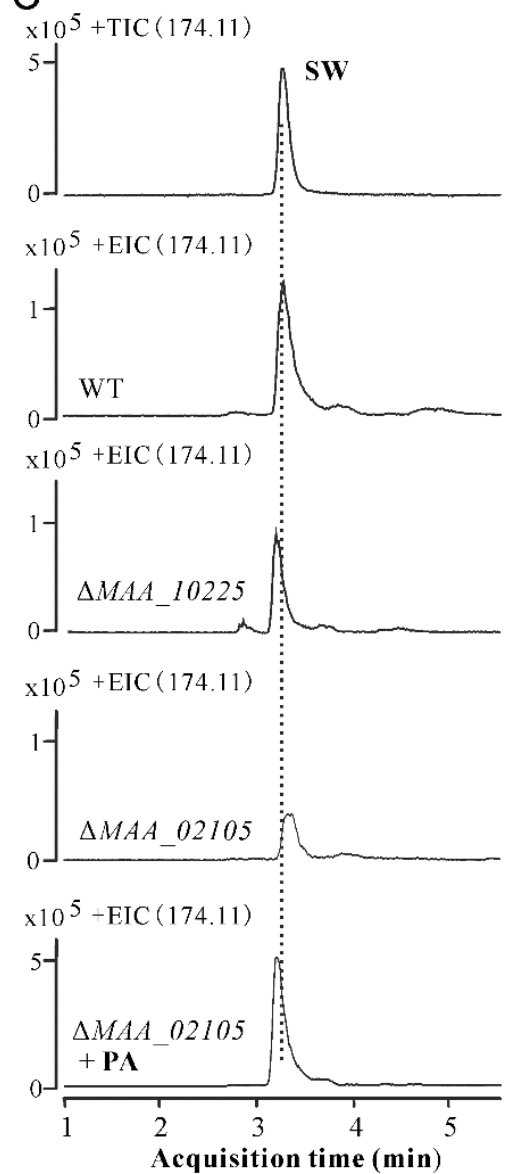

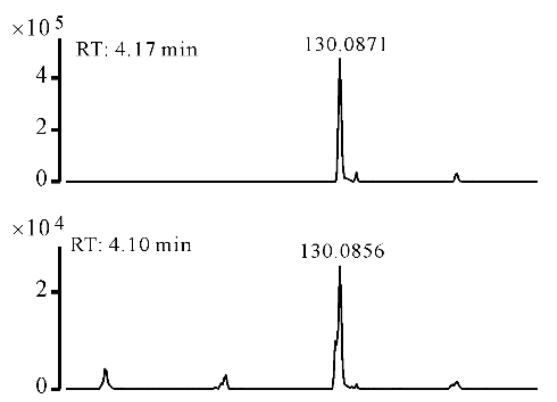

$\times 10^{5}$ RT: $4.01 \mathrm{~min} \quad 130.0867$

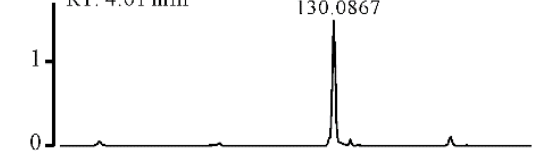

$\times 10^{5}$ RT: $4.13 \mathrm{~min} \quad 130.0862$

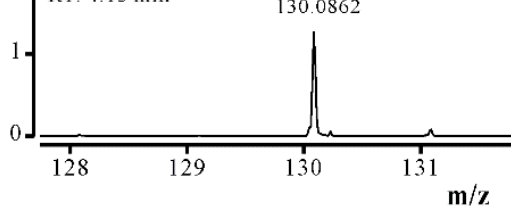

D
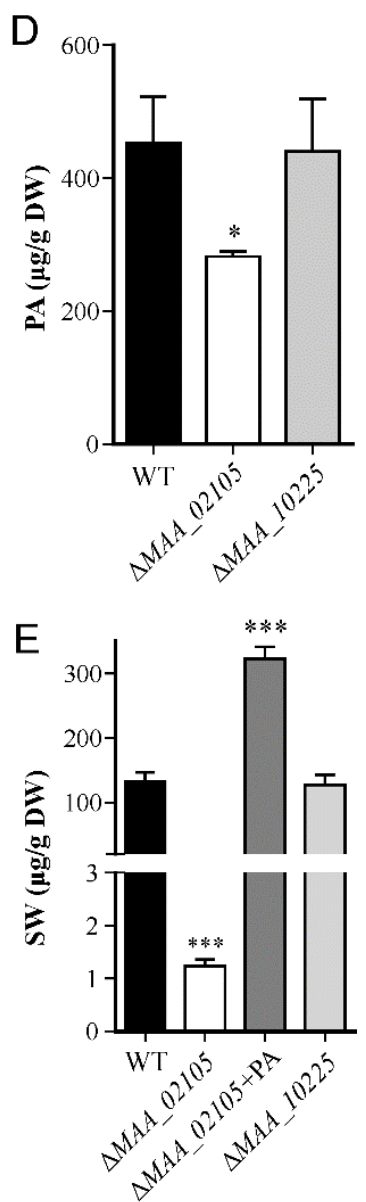

Figure S2. Functional verification of genes involved in the production of pipecolic acid (PA) and swainsonine (SW). (A) LC-MS analysis of PA production by the transgenic yeast cells. The yeast cells transformed respectively with an empty vector (Blank + Lys), and swnA (SwnA + Lys) or both swnA and swnR (SwnA.R + Lys) genes were fed with Lys at a final concentration of $10 \mathrm{mM}$. The right panels show the extracted ion chromatogram (EIC 
of PA standard and different treatments); the left panels show the $\mathrm{m} / \mathrm{z}$ values of the corresponding peaks detected in EIC analysis. (B) LC-MS analysis of PA production in different strains of $M$. robertsii and B. bassiana. (C) LC-MS analysis of SW production in different strains of $M$. robertsii. For each strain, the same amount of the freeze dried mycelia was used for metabolite extraction and LC-MS analysis. (D) Comparative analysis of PA production in the WT and different mutants of $M$. robertsii. Each column represents the value of mean \pm SE. The degree of $t$-test difference between WT and individual mutant is indicated by $*, P<0.05$. (E) Comparative analysis of SW production in the WT and different mutants with and without the supplementation of PA. Each column represents the value of mean \pm SE. The degree of difference between WT and individual mutant is indicated by $* * *$, $P<0.001$. EIC, extracted ion chromatogram. 
A

ErYKR1 DDEWKPTGTVLVTGGTGGVGGQIARWLARR - GAPHLLLVSRSGPDADGAGELVAELEALGARTTVAACDV NYSKR1 ARTWAPRGTTLITGGSGTLAPOI.ARWLAER GAEHVVLVSRRGADAPGAPELIAEAAESGTEVTVAACDI RifKR7 PLTFRPDGTVLVS-GAGTLGALAARDLVTRHGVRRLVLASRRGRAAEGIDDLVAELTGHGAEVTVAACDV RifKR10 AAGFKTRGPVLVTGGTGSLGGLVARHLVERHGVRQLVLASRRGLDAEGAKDLVTDLTALGADVAVAACDV PICKR3 PSGLDPEGTVLLTGGTGALGGIVARHVVGEWGVRRLILVSRRGTDAPGAGELVHELEALGADVSVAACDV SWIK-KR QPFVRTDGAVLVTGGLGDLGSRVARRLATAHGVCDLVLVSRQGTNSPGADALVAELAELGAKATIVGCDL HPm8-KR SLKLDPDATYLFVGGLGGLGRSLAKEFVAS-GARNIAFLSRSGDTTAQAKAIVDELAGQGIQVKAYRGDI Rdc5-KR SFKLDPNATYLFIGGLGGLGRSMAVGFVAC-GARNIAFLSRSGDSKPEAKAVVDELRELGTRVQVYLGDV

ErYKR1 NYsKR1

RifKR10

PicKR3

SWIK-KR

Hpm8 - KR

Rdc5-KR

TDRESVRELLGGIGDD-VPLSAVFHAAATLDDGTVDTLTGERIERASRAKVLGARNLHELTR--ELDLTA TDRDAVAALIADLTADGRTLRTVIHAAAAIELSALADTTVAEFADVVHAKVTGARILDELLD DAELDD SDRDQVAALLK E HALTAVVHTAGVFDAGVTGALTRERLAKVFAPKVDAANHLDELTR DLDLDA ADRDOVAALIT - - - E-HRP SAVVHTAGVPDAGVIGTVTPDRLAEVFAPKVTAARHLDELTR - DLDLDS ADREALTAVLDSIPAE - HPLTAVVHTAGVLSDGTLPSMTAEDVEHVLRPKVDAAFLLDELTSTPGYDLAA ADLDSLGSVMOLFTPD-RPLRGVVHAAGVVDSGTLSSLTPRKCAAVFAPKVDGLWNLHOLTK - HMDLDL ASEASFLQAMEQC SQDLPPVRGVIQMAMVLRDIVFEKMSYDEWTVPVGPKVQGSWNLHKYFSH-ERPLDF SDEASFRGAMEQCSRELPPVKGVIQMAMVLRDVVFEKMKYDDWTTGLRPKVQGTWNLHTFFDK-DRPLDF : $\quad: \quad:$ : . $.4 * *$ * : ErYKR1 FVLFSSFASAFGAPGLGGYAPGNAYLDGLAQQRRSDGLPATAVAWGTWA- - - - GSGMAEGAVADRFRRH NYSKR1 FVLYSSTAGMWGSGVHAAYVAGNAYLSALAEQRRARGLRTTSIHWGKWP DDRARELADPHRIRRS RifKR7 FIVYSSASSIFMGAGSGGYAAANAYLDGLMAARRAAGLPGLSLAWGPWEOLTG-MADTIDDLTLARMSRR Ri fKR10 FVVYSSVSAVFMGAGSGSYAAANAYLDGLMAHRRAAGLPGOSLAWGLWDOTTGGMAAGTDEAGRARMTRR

PickR3

SWRK-KR

Hpm8-KR FVMFSSAAAVFGGAGOGAYAAANATLDALAWRRRTAGLPALSLGWGLWAETSG-MTGGLSDTDRSRLARS FMMFSSISGIIGLPGLGNYAAANSFIDSLAHLRRAQGLPASSVAYGTWA-GDG-MATTLVPTTRAHLSOL MVICSSSSGIYGYPSQAQYAAGNTYQDALAHYRRSQGLNAI SVNLGIMR - - -DVGVLAETGTTGNIKLW MIFFSSIAGVFGNPSQAQYAAGNTYQDSLAKYRRDRGLKAVSVNLGIMR-- - -DVGVIAE-GDSHFMQQW

RdC5-KR

$: * 4:$

$$
\text { - } 4 \cdots 4: \ldots * * * * \text { * : : }
$$

ErYKR1

NYsKR1

RifKR7

RifKR10

Pickro

SWnK-KR

Hpm8 $-\mathrm{KR}$
$\mathrm{Rdc5}-\mathrm{KR}$

B

- - - -GVIEMPPETACRALONALDRAEVCPIVIDVRWDRFLLAYTAORPTRLFDEIDDAR - - -

- - - -GLEYLDPELALTALQHVLDDDETVIGLMDIDWDTYHDVFTAGRPAHLFDQIPEVRRRLD

EGRGGVRALGSADGMELFDAALAAGQALLVPIELDLREVRADAAGGGTVPHLIRGLVRAGRQA

- - GGLVAMKPAAGLDLFDAAIGSGEPLLVPAQLDLRGLRAEAAGGTEVPHLLRGLVRAGRQQA

- - - GATPMDSELTLSLLDAAMRRDDPALVPIALDVAALRAQQRDGMLAP-LLSGLTRGSR - -

- - GLGFLPPEAGLEI FEHAVYOGRALTVAAVLDLORLRAYYEEOGGVPPLLNSMLGGTKVOK

EEVLGIREPAFHALMKSLINHQQRGS - - - - GDYPAQVCTGLGTADIMATHGLAARPEYFNDPRF

EEVLGIREPAFHALIKSIINGQLETSNIREAAKCPVQVTVGLGTGDILARNKIREPDYFRDPRF

- -RTILLTGATGFLGAFLLEELCRRTDARIYCLVRSKTEOEGMNRIRKNLESYSLWNEALAPRIVPVRGD - - - SVLITGATGFL GAYLLYELLKOTDANVFCLVRSNHSEAAYORIHSTLKFYOLWSESWRSRIIPVCGD - - RHILITGATGFLGSFLLRDLLRSTSARIHCLVRGADASDARARLTAAAAWYETGADLDFDRIDIAVGD - - - -VLLTGATGFL GSHLLDELORAG-RRVCCLVRAGSVEEARGRLRAAFEKFALDPSR-LDRAEIWLGD - - - VLLTGATGFL GSHLLDELQRAG-RRVCCLVRAGSVEEARGRLRAAFEKFALDPSR-LDRAEIWLGD
NPRRVFLTGATGYLGLHLVEOLLRRTDAEVVTLCRARDEHALERLKEGFALYEIDVEDQLHRISAVIGD - - TVFVTGATGFVGTFMIHEFLORR - ISVYCLVRAS GSQFAQQRMITTLKQYGLWRPEHEPLLVSVAGD SGLEILLTGATGFL GGQLLERLVQS PRVSTVHCVAVPVDEQSLLEPFLQQQADGT - - - - RRKVRCY I RN

- -AVVVLTGATGFL GRAIVNRLLKDCSVOKI HCVAVRRDPSSLPDDFKSP - - - - - - - - KVVLHRGD -NLTIVLAGATGYLGRHILARLVEAPEIGEIHCLVRK - -ESLEMTDRSS - - - - - - - - LKVRLVTAD

$$
::: * * * *:: * \quad:: \quad: \text { : }
$$
ISOPSLGL SAEEFSKLTELIDAIYHNGAOVSAIEPYTYLKPTNVLGTSELLDFAARCRVKPLHFVSTAAV LAEPALGLDEAAFDELARNVDVVYHAGASVNWLYPYEALRPANIAGTEEVLRLAARHRTVPVHYISSTGV LARPRLGLGDG - FAARAHEVGEVYHAAAHINFAVPYHTVKRTNVDGLRRVLDFCGVNRT - PLRLISTLGV LAEPRLGLTOEOWDDLAATVDVIYHNGALVNFVYPYSALKAANVGGTQRVLELACTTRLKAVHHVSTIDT LSOPLLGLGEVVFDDLASRVDAILHSGALVDWMRPLDDYIGPNVLGTHEVLRLASCGRAKAIHFISTIST LAAPALGLTAADOTALSOTADVIVHAGSMGHCLNTYATLAAPNFASTRHLCSLALSRSPPIPLAFASSNR LTLPOLGLTDRAATEIFAEADAVIHNGADVSFMKTYOSLKOANLEATKELVRLSAPHR - -LSFHYISSAS LTQPRLGLTEGEFIALAEKTDVIVNCAANRSFWDGYDTLRTVNFDAVKELVTLSVRAGG-APLHTVSSGA : **** $\quad$ : : : : $\quad$ *. . : :

MXaA-R

LtXA-R

CycB-R

Orf22-R

SfmC-R

SwnK-R

Tens-R

Cons

CCSA-R
EqiS-R

LPLGRKAP - -IREDEPLEGPSSLVGGYAQSKWVAEKLVREASRRGL AVSSKGNPDIIYENFRLGADSVLPSGYVSSKWVAERLVWVASDRGL YAQEPAEGRRIAVDDPIGPPELLSNGYRQAKWVAEGIIGIARSRGI FPPDSAPG - VIGEDTVPGDPASLGIGYSQSKWVAEHLALQARQAGL LLATHMPRPFLENDAPLHSAVGVPAGYTGSKWVAEKVVDEARRRGI LPIHAGYGLAEHDG - - - - - - - EYGYGTSKYLAEKMVVAARFRGA VALLTGSTAPPPASAAAFPPPPGAOGFTASKWASEAFLEKILAASTM

\begin{tabular}{|c|c|c|}
\hline 192 & NRPS & \\
\hline 193 & NRPS & 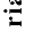 \\
\hline 194 & PKS & \\
\hline 195 & NRPS & 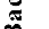 \\
\hline 196 & NRPS & \\
\hline 173 & NRPS/PKS & \\
\hline 181 & PKS/NRPS & \\
\hline 169 & PKS/NRPS & $=$ \\
\hline 160 & PKS/NRPS & \\
\hline
\end{tabular}
VDVYGDNK - - - - - - - PPVEGSDGYVASKWSTERFLANAWKELG 169 PKS/NRPS

Figure S3. Sequence alignments of the conserved fungal and bacterial KR and $K$ domains. (A) Alignment of the KR domains known or predicted to show stereocatalytic activity. Consistent with the characterized catalytic residues $\mathrm{K}, \mathrm{S}, \mathrm{Y}$ and $\mathrm{N}$ identified in Hpm8 (arrowed), the conserved tetrad is also present in SwnK and other sequences. In addition, the conserved NADPH/NADH binding sites (framed) are similarly present in different sequences. Ery, erythromycin synthase (protein ID: Q03131); Nys, nystatin synthase (AAF71775); Rif, rifamycin synthases (YP_003762841 for RifKR7 and YP_003762843 for RifKR10); Hpm8, hypothemycin synthase (B3FWT3); Rdc5, radicicol 
synthase (B3FWU0). (B) Alignment of the R domains known or predicted to mediate reductive cleavage. MxaA, myxalamid synthetase (AAK57184); LtxA, lyngbyatoxin synthetase (AAT12283); CycB, Cyclizidine synthase (ALP32042); Orf22, benzodiazepine synthetase (ABW71853); SfmC, saframycin synthetase (ABI22133); TenS, tenellin synthase (CAL69597); CcsA, cytochalasin synthase (ACLA_078660); and EqiS, equisetin synthase (ACLA_023380). The conserved NADPH/NADH binding sites are framed, and the characterized catalytic residues are arrowed. 


\section{A}
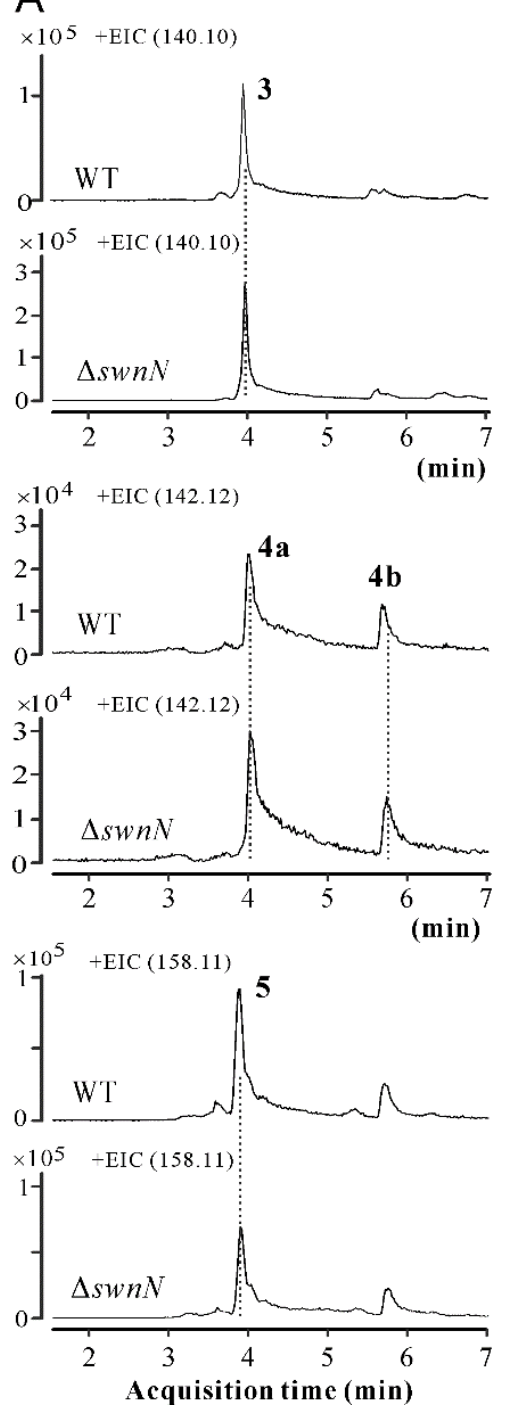

B
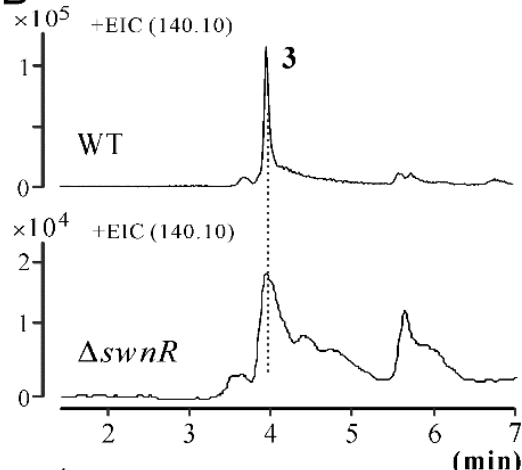

$\times 10^{4}+$ EIC $(142.12)$
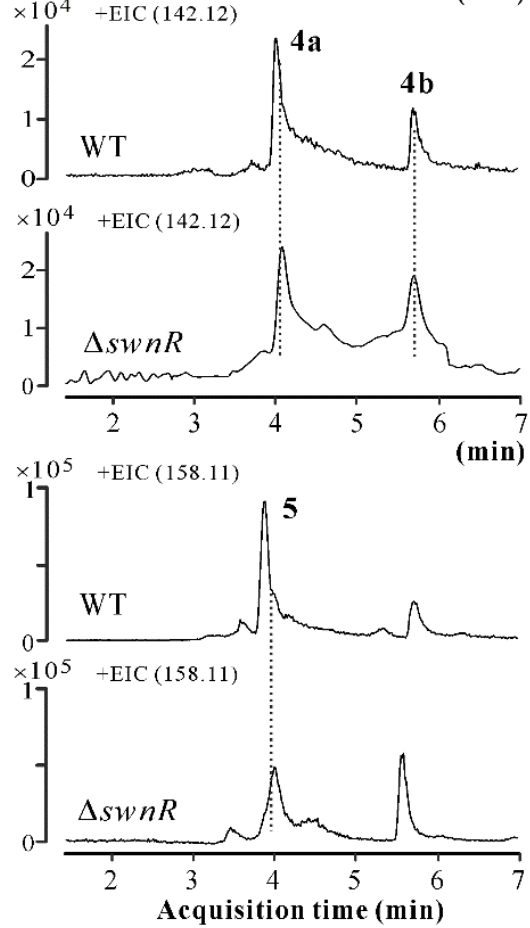

C
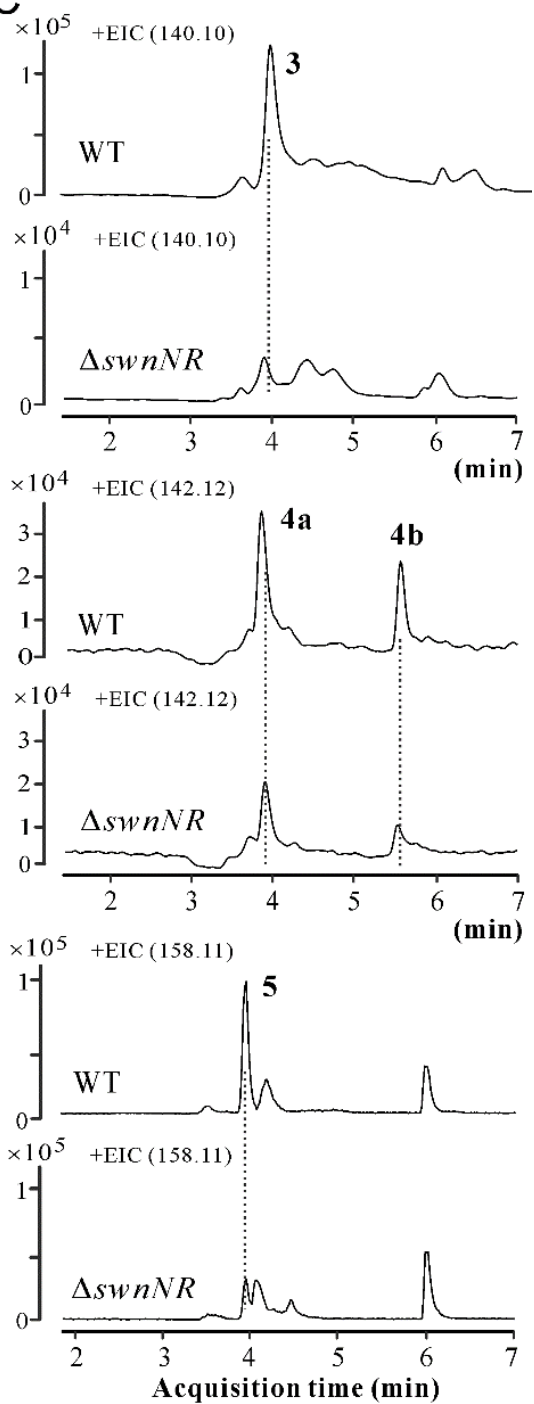

Figure S4. The effects of $s w n N$ and $s w n R$ on the production of different intermediates.

(A) Comparative LC-MS analysis of different intermediates produced by the wild type (WT) and $\Delta s w n N$. (B) Comparative LC-MS analysis of different intermediates produced by the WT and $\Delta s w n R$. (C) Comparative LC-MS analysis of different intermediates produced by the WT and $\Delta s w n N R$. For each strain, the same amount of the freeze dried mycelia was used for metabolite isolation and LC-MS analysis. EIC, extracted ion chromatogram. The WT and mutants are labeled at the bottom left of each panel. 


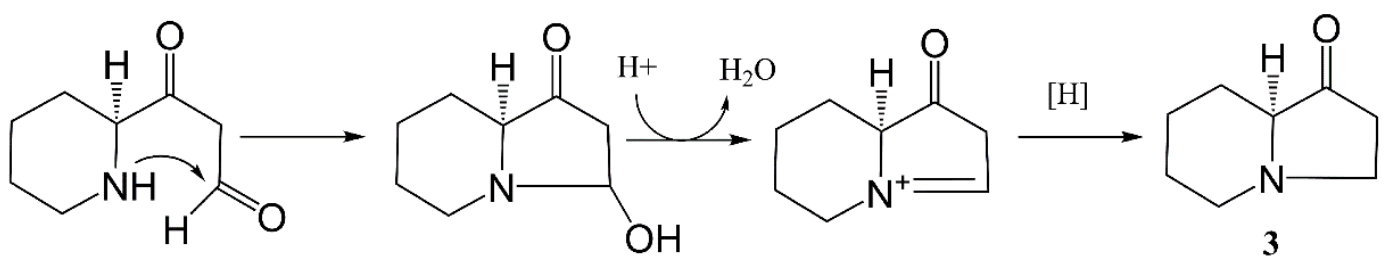

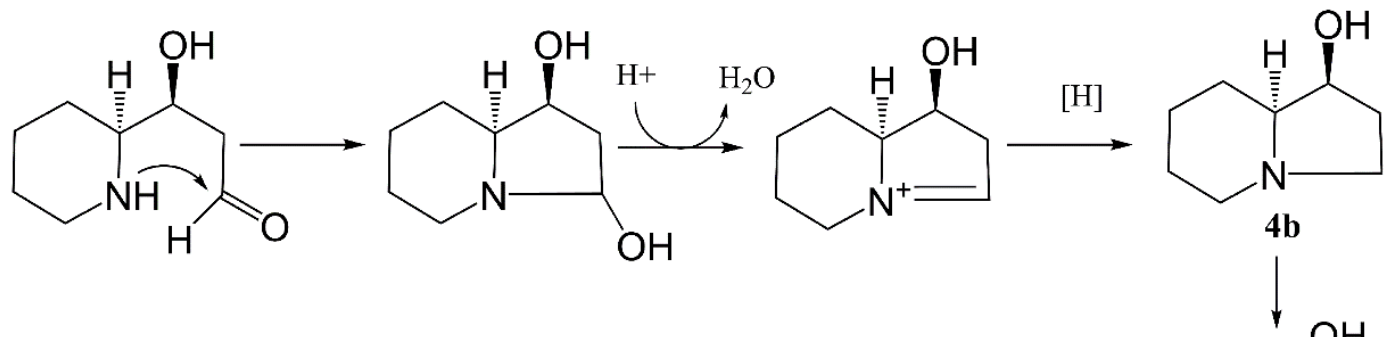

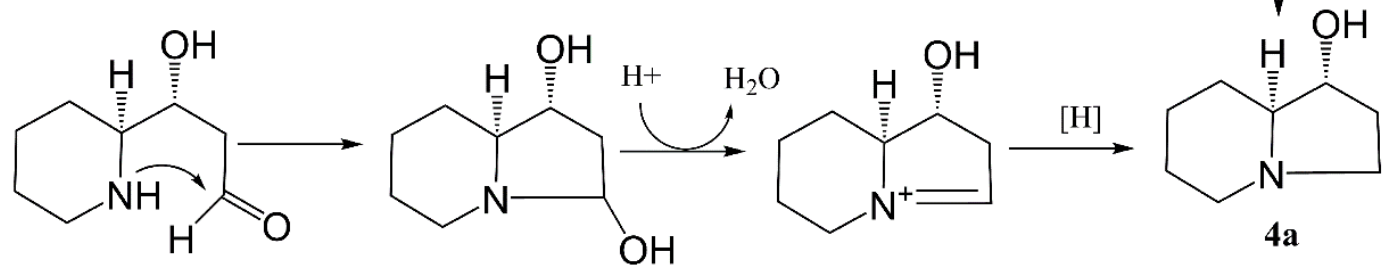

Figure S5. Schematic pathways for the conversion of compounds 3 and $4 a / b$ through the formation and reduction of iminium ions. 

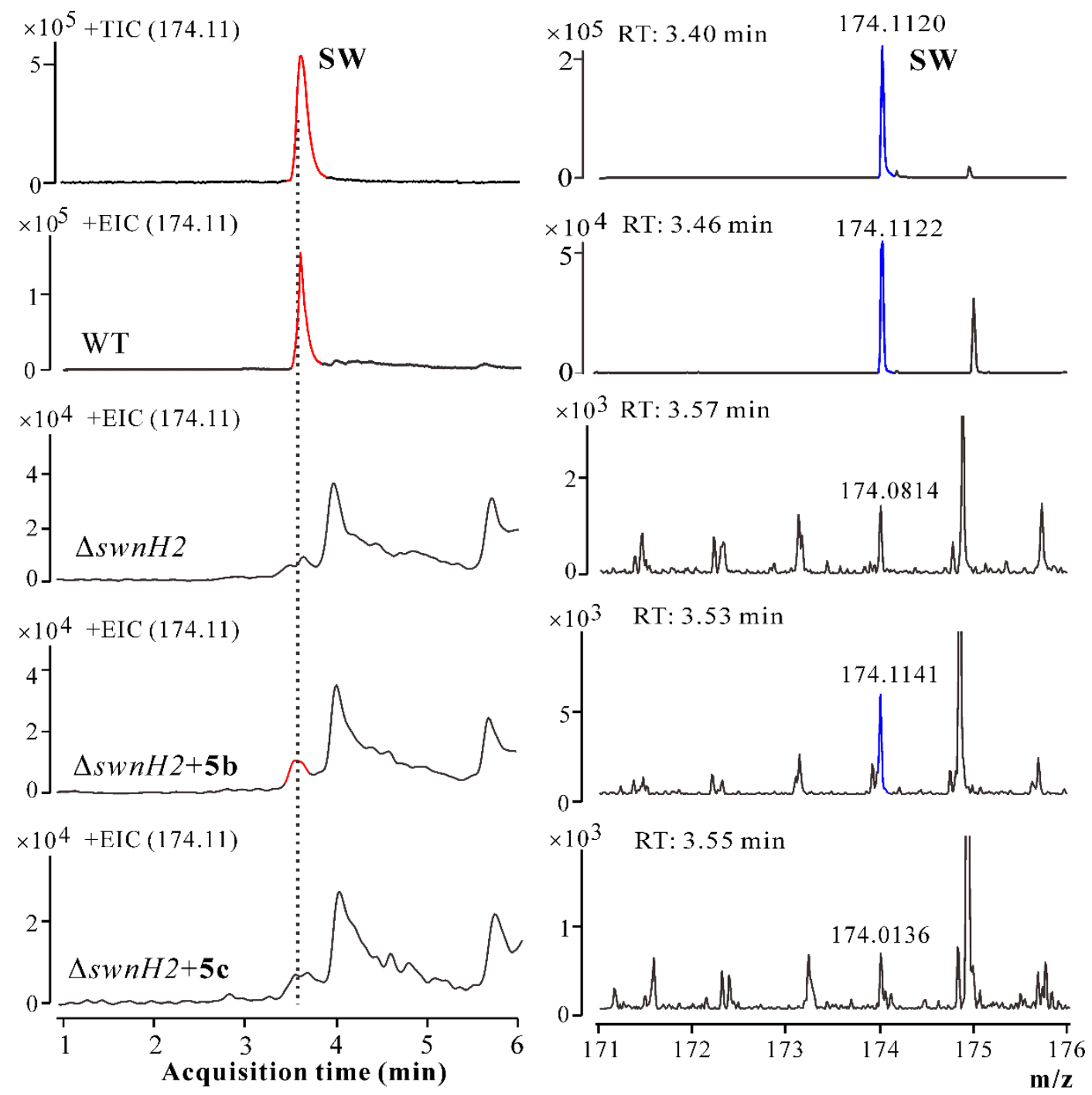

Figure S6. LC-MS analysis of SW production during $5 b$ and $5 c$ feeding assays. The spores of $\triangle s w n H 2$ were inoculated in SDB for four days, and $\mathbf{5 b}$ or $\mathbf{5} \mathbf{c}$ was then supplemented at a final concentration of $1 \mathrm{mM}$ for another three days. Together with the WT samples, the mycelia were extracted for LC-MS analysis of SW production. The left panels show the swainsonine peak in the extracted ion chromatogram (EIC; red); the right panels show the mass spectra of detected SW (blue peak). The WT and mutants are labeled at the bottom left of each EIC panel. 
A

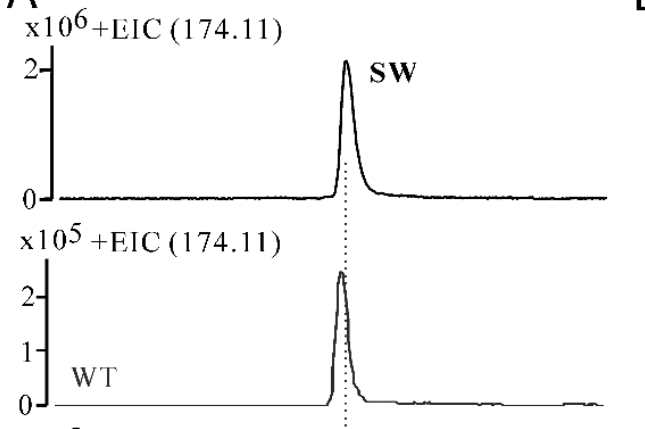

$\times 10^{5}+\mathrm{EIC}(174.11)$

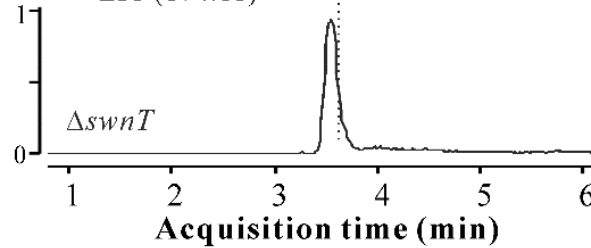

C
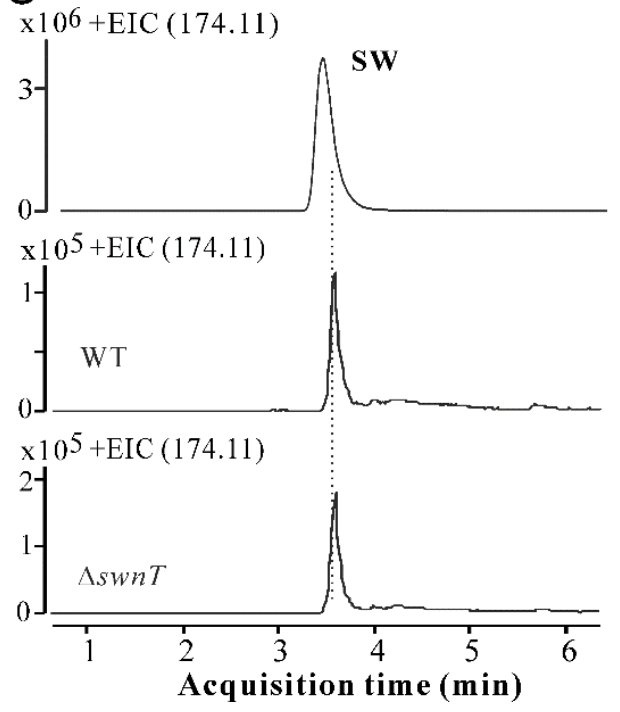

B

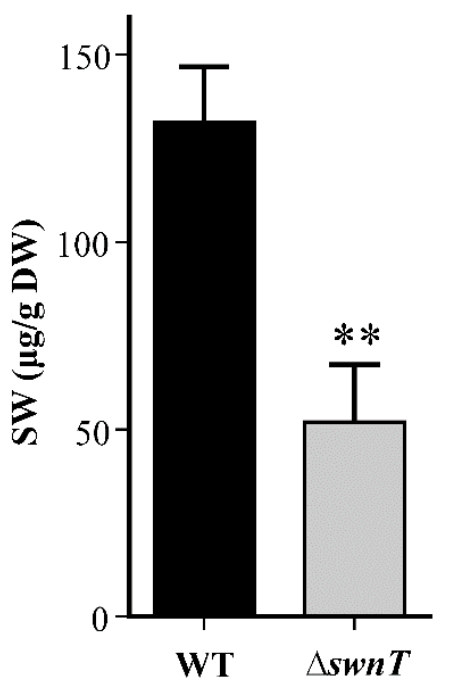

$\mathrm{D}$

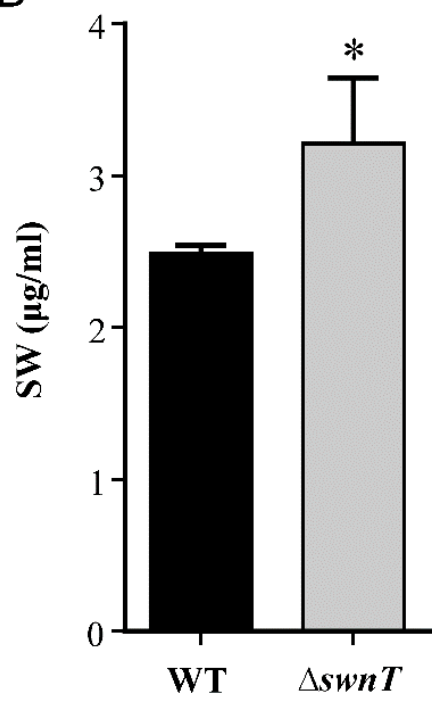

Figure S7. Examination of SwnT to the intracellular and extracellular accumulation of swainsonine (SW). (A) LC-MS analysis of SW extracted from the mycelia. (B) Comparative analysis of the intracellular accumulation of SW between wild type (WT) and $\Delta s w n T$. DW, dry weight. (C) LC-MS analysis of SW extracted from the culture filtrates. (D) Comparative analysis of SW accumulation in culture filtrates between WT and $\Delta s w n T$. The fungi were incubated in SDB at $30{ }^{\circ} \mathrm{C}$ and $200 \mathrm{rpm}$ for seven days for SW extraction from the harvested mycelia and culture filtrates. Each column represents the value of mean $\pm \mathrm{SE}$. The degree of difference between WT and individual mutant is indicated by **, $P<0.01$; , $P<0.05$. EIC, extracted ion chromatogram. The WT and mutants are labeled at the bottom left of each panel. 

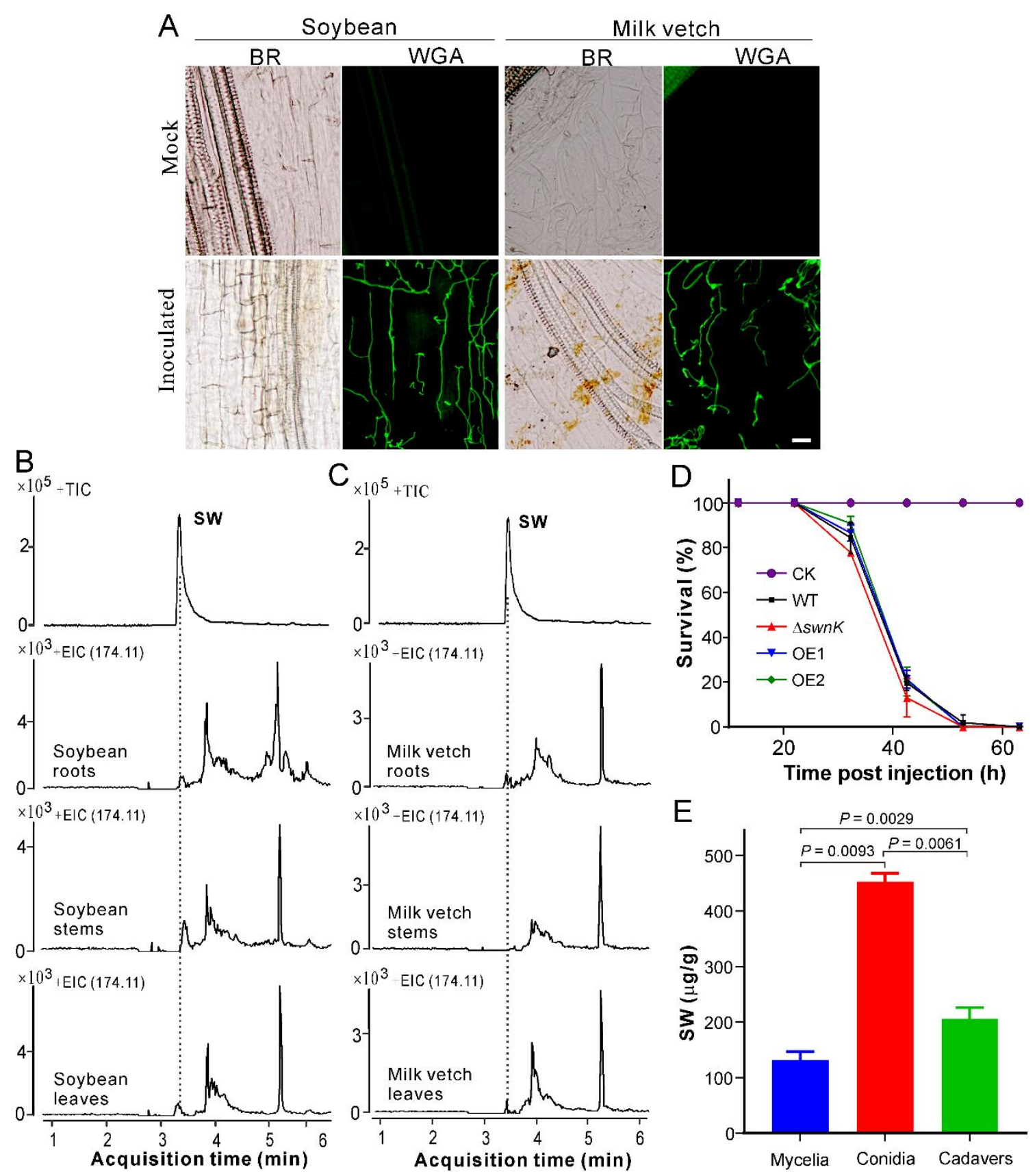

Figure S8. Plant root colonization and insect assays. (A) Plant root colonization assays of soybean (Glycine max) and milk vetch (Astragalus sinicus) by the WT strain. Plant seeds were surface sterilized and inoculated with the spores of the WT strain of M. robertsii for germination and growth for up to 30 days. Plant roots were then dissected for lectin staining. The un-inoculated plants were grown in parallel as a mock control. BR, bright field; WGA, wheat germ agglutinin staining. Bar, $30 \mu \mathrm{m}$. (B) LC-MS analysis indicates that swainsonine (SW) could not be detected from soybean roots, stems and leaves after the plant being inoculated with fungal spores for 30 days. (C) LC-MS analysis indicates that SW could not be detected from milk vetch roots, stems and leaves after the plant being inoculated with fungal spores for 30 days. (D) Insect survivals. Wax moth larvae were individually injected with the spores of the WT and different mutants for survival assays. Control insects (CK) 
were treated with $0.05 \%$ Tween-20. OE1 and OE2, two independent swnA overexpression mutants. (E) SW production by the WT strain grown in different media. Mycelia: mycelia harvested from SDB for one week; Conidia: conidial spores harvested from two-week old PDA plates; Cadavers: Mycosed insect cadavers. All samples were freeze dried and weighted before use for SW extraction. Each column represents the value of mean $\pm \mathrm{SE}$. 


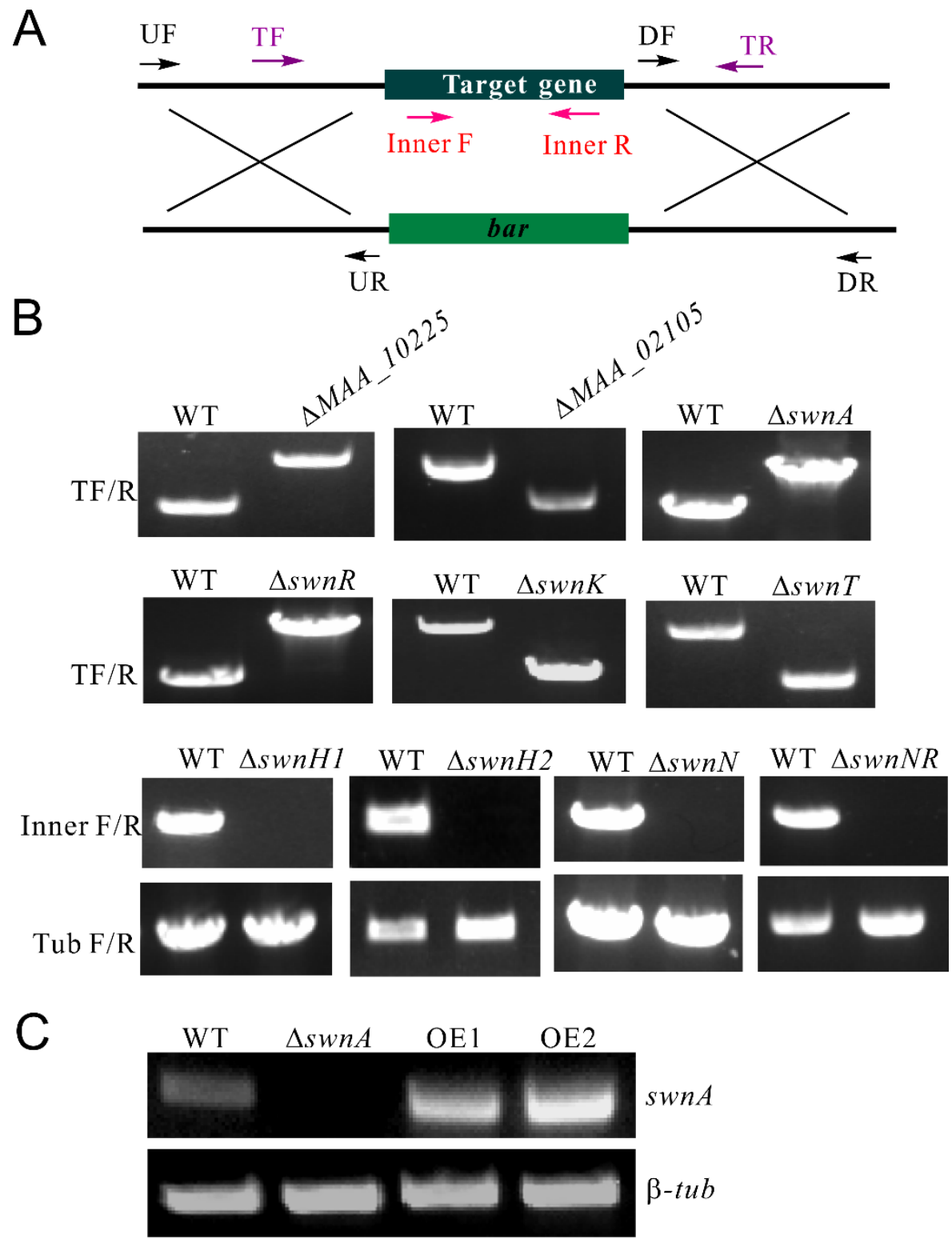

Figure S9. Gene deletion, overexpression and verification. (A) A schematic map for the homologous replacement of gene deletion. The genomic DNA was used as a template and the primer pairs UF/UR and DF/DR are used to amplify the flanking regions of the target gene while the primers TF/TR and innerF/R are used for verifications. (B) PCR verification of gene deletions. Both the primer pairs TF/TR and inner F/R were used for verification for all or selected mutants. Tub, a $\beta$-tubulin gene was amplified as a reference. (C) RT-PCR verification of swnA overexpression. The fungi were grown in SDB for one weeks and RNA was extracted from each sample for analysis. 
Table S1. NMR data collected for the compound 3 formate in $\mathrm{CDCl}_{3}$ and 4 a formate in $\mathrm{CD}_{3} \mathrm{OD}$.

\begin{tabular}{|c|c|c|c|c|c|}
\hline \multirow{2}{*}{ Position } & \multicolumn{2}{|c|}{3 formate } & \multicolumn{3}{|c|}{ 4a formate } \\
\hline & $\delta_{\mathrm{H}}(J$ in $\mathrm{Hz})$ & Calculated $\delta_{\mathrm{H}}$ & $\delta_{\mathrm{C}}$ & $\delta_{\mathrm{H}}(J$ in $\mathrm{Hz})$ & Calculated $\delta_{\mathrm{H}}$ \\
\hline 1 & & & 74.1 & $\begin{array}{l}4.09, \mathrm{ddd} \\
(8.4,7.2,4.6)\end{array}$ & 4.36 \\
\hline \multirow[t]{2}{*}{2} & $3.15, \mathrm{~m}$ & 2.94 & 31.4 & $2.37, \mathrm{~m}$ & 2.33 \\
\hline & $3.03, \mathrm{~d}(11.8)$ & 2.79 & & $1.81, \mathrm{~m}$ & 1.95 \\
\hline \multirow[t]{2}{*}{3} & $4.12, \mathrm{~m}$ & 4.03 & 51.4 & $3.41, \mathrm{~m}$ & 3.49 \\
\hline & $3.59, \mathrm{~m}$ & 3.36 & & $3.09, \mathrm{~m}$ & 3.24 \\
\hline \multirow[t]{2}{*}{5} & $4.05, \mathrm{~m}$ & 3.73 & 52.2 & $3.41, \mathrm{~m}$ & 3.60 \\
\hline & $3.21, \mathrm{~m}$ & 3.36 & & $2.85, \mathrm{~m}$ & 2.98 \\
\hline \multirow[t]{2}{*}{6} & $1.53, \mathrm{~d}(7.0)$ & 2.01 & 23.2 & $1.81, \mathrm{~m}$ & 1.98 \\
\hline & & 2.01 & & $1.72, \mathrm{~m}$ & 1.69 \\
\hline \multirow[t]{2}{*}{7} & $1.86, \mathrm{~m}$ & 2.10 & 23.1 & $1.89, \mathrm{~m}$ & 1.98 \\
\hline & $1.48, \mathrm{~m}$ & 1.62 & & $1.49, \mathrm{~m}$ & 1.61 \\
\hline \multirow[t]{2}{*}{8} & $1.86, \mathrm{~m}$ & 2.35 & 26.4 & $2.08, \mathrm{~m}$ & 2.33 \\
\hline & $1.68, \mathrm{~m}$ & 2.01 & & $1.49, \mathrm{~m}$ & 1.69 \\
\hline $8 \mathrm{a}$ & $3.73, \mathrm{~m}$ & 3.58 & 71.0 & $2.79, \mathrm{~m}$ & 2.91 \\
\hline
\end{tabular}


Table S2. NMR data collected for the compounds $5 \mathrm{~b}$ and $5 \mathrm{c}$ formates in $\mathrm{CD}_{3} \mathrm{OD}$.

\begin{tabular}{|c|c|c|c|c|c|}
\hline \multirow{2}{*}{ Position } & \multicolumn{3}{|c|}{$\mathbf{5 b}$ formate } & \multicolumn{2}{|c|}{$\mathbf{5 c}$ formate } \\
\hline & $\delta_{\mathrm{C}}$ & $\delta_{\mathrm{H}}(J$ in $\mathrm{Hz})$ & Calculated $\delta_{\mathrm{H}}$ & $\delta_{\mathrm{H}}(J$ in $\mathrm{Hz})$ & Calculated $\delta_{\mathrm{H}}$ \\
\hline 1 & 74.5 & $3.87, \mathrm{dd}(9.3,6.1)$ & 4.09 & 3.80, dd $(8.6,3.6)$ & 4.10 \\
\hline 2 & 67.8 & $\begin{array}{l}4.28, \text { ddd } \\
(6.9,6.1,4.3)\end{array}$ & 4.27 & $\begin{array}{l}\text { 4.11, ddd } \\
(7.3,3.2,2.3)\end{array}$ & 4.24 \\
\hline 3 & 60.6 & $\begin{array}{l}3.83, \mathrm{dd}(12.0,6.7) \\
2.82, \mathrm{~m}\end{array}$ & $\begin{array}{l}3.87 \\
3.10\end{array}$ & $\begin{array}{l}3.14, \mathrm{dd}(11.7,2.1) \\
3.07, \mathrm{dd}(11.6,7.4)\end{array}$ & $\begin{array}{l}3.43 \\
3.28\end{array}$ \\
\hline 5 & 53.4 & $\begin{array}{l}3.41, \mathrm{~m} \\
2.82, \mathrm{~m}\end{array}$ & $\begin{array}{l}3.59 \\
3.01\end{array}$ & $\begin{array}{l}3.42, \mathrm{~m} \\
2.55, \mathrm{~m}\end{array}$ & $\begin{array}{l}3.57 \\
3.03\end{array}$ \\
\hline 6 & 24.2 & $\begin{array}{l}1.83, \mathrm{~m} \\
1.74, \mathrm{~m}\end{array}$ & $\begin{array}{l}2.02 \\
1.76\end{array}$ & $\begin{array}{l}1.77, \mathrm{~m} \\
1.70, \mathrm{~m}\end{array}$ & $\begin{array}{l}1.99 \\
1.74\end{array}$ \\
\hline 7 & 23.1 & $\begin{array}{l}1.90, \mathrm{~m} \\
1.55, \mathrm{~m}\end{array}$ & $\begin{array}{l}1.93 \\
1.58\end{array}$ & $\begin{array}{l}1.88, \mathrm{~m} \\
1.42, \mathrm{~m}\end{array}$ & $\begin{array}{l}1.99 \\
1.64\end{array}$ \\
\hline 8 & 26.9 & $\begin{array}{l}2.11, \mathrm{~m} \\
1.55, \mathrm{~m}\end{array}$ & $\begin{array}{l}2.31 \\
1.58\end{array}$ & $\begin{array}{l}2.05, \mathrm{~m} \\
1.52, \mathrm{~m}\end{array}$ & $\begin{array}{l}2.37 \\
1.74\end{array}$ \\
\hline $8 \mathrm{a}$ & 67.7 & $2.92, \mathrm{~m}$ & 3.34 & $2.55, \mathrm{~m}$ & 2.91 \\
\hline
\end{tabular}


Table S3. PCR primers used in this study.

\begin{tabular}{|c|c|c|c|c|}
\hline Gene & Primers & Sequence & Restriction & Application \\
\hline \multirow{6}{*}{$\begin{array}{l}\text { MAA_ } \\
10225\end{array}$} & UF & CGGGATCCGCTGATTGGGTTCATACT & Bam HI & \multirow{4}{*}{ Gene deletion } \\
\hline & UR & CGGGATCCAGCGAGCACAACGACTAA & Bam HI & \\
\hline & DF & GACTAGTTGACGGAATGACGACAGG & SpeI & \\
\hline & DR & GACTAGTGTATCGCCACAAGGTGCA & SpeI & \\
\hline & TF & GGCTTTCGTTACATTCCC & & \multirow{2}{*}{ PCR verification } \\
\hline & TR & CTCCGAGTCTTATTCACCAC & & \\
\hline \multirow{6}{*}{$\begin{array}{l}M A A_{-} \\
02105\end{array}$} & UF & GGAATTCCTGGGAGATTCAGGGTGC & Eco RI & \multirow{4}{*}{ Gene deletion } \\
\hline & UR & TCCCCCGGGCGGGCTCAATGACAATACA & SmaI & \\
\hline & DF & GACTAGT CAAGGAGCGGGAAGGATG & SpeI & \\
\hline & DR & GACTAGT GTTCGGAAAGGAAGTGGG & SpeI & \\
\hline & $\mathrm{TF}$ & GGTGCCCAAATAGCTTAC & & \multirow{2}{*}{ PCR verification } \\
\hline & TR & ATGACGATGGTGCTTCTC & & \\
\hline \multirow{10}{*}{ SwnA } & UF & GGAATTCAGCCCAAACTCGTCCCTCAC & Eco RI & \multirow{4}{*}{ Gene deletion } \\
\hline & UR & AACTGCAGGGACTGGGACACGACGCTGA & Pst I & \\
\hline & DF & GACTAGTCTATCAACCACCCAACAGC & Spe I & \\
\hline & DR & CGAGCTCGCGGAGTAGGGAGGAAAT & Sac I & \\
\hline & $\mathrm{TF}$ & AAAAGTACGGGTCGTCCTCG & & \multirow{2}{*}{ PCR verification } \\
\hline & TR & CGTCTGTCTGGGTCGGTGA & & \\
\hline & Hsp70F & GCTCTAGAATGCACTTGGAGCGGGACAAG & Xba I & \multirow{2}{*}{ Overexpression } \\
\hline & Hsp70R & CGAGCTCTCACCCCAGCCCAAACTCGTCCC & Sac I & \\
\hline & Sc-ExF & CGAGCTCATGCACTTGGAGCGGGACAA & Sac I & \multirow{2}{*}{ For yeast expressior } \\
\hline & Sc-ExR & CGAGCTCTCACCCCAGCCCAAACTCGT & Sac I & \\
\hline \multirow{6}{*}{ swnH1 } & UF & CCGCTCGAGTGCGACCGAACAATGACA & Xho I & \multirow{4}{*}{ Gene deletion } \\
\hline & UR & GGAATTCGCTGTGCGAATCAAGTGC & Eco RI & \\
\hline & DF & GACTAGTAAGTGCTGGGGATGAGTG & Spe I & \\
\hline & DR & CGAGCTCCATGTTTCTGTGGGTGGG & Sac I & \\
\hline & Inner F & CGACATCATCGCCACCCT & & \multirow{2}{*}{ PCR verification } \\
\hline & Inner R & TGGCCAGAAACTGATTCTCCTC & & \\
\hline \multirow{6}{*}{$\operatorname{swn} T$} & UF & CCGCTCGAGATACGACTCGGTGGAATG & Xho I & \multirow{4}{*}{ Gene deletion } \\
\hline & UR & GGAATTCGCCCTGTTTGGTTGTTTG & EcoR I & \\
\hline & DF & GACTAGT TGCGACCGAACAATGACA & Spe I & \\
\hline & DR & CGAGCTCGCTGTGCGAATCAAGTGC & Sac I & \\
\hline & TF & ATCCGTGACTGAATACCAACT & & \multirow{2}{*}{ PCR verification } \\
\hline & TR & AAGGTAATCCACCCAAAGG & & \\
\hline \multirow{6}{*}{$\operatorname{swnN}$} & UF & CCGCTCGAGACAGCAATGAGACGTCGGG & Xho I & \multirow{4}{*}{ Gene deletion } \\
\hline & UR & GGAATTCCAGATCGTGGCGGCACTT & EcoR I & \\
\hline & DF & GACTAGTATACGACTCGGTGGAATG & Spe I & \\
\hline & DR & CGAGCTCGCCCTGTTTGGTTGTTTG & Sac I & \\
\hline & Inner F & ACCGTGCTGGATGCTATT & & \multirow{2}{*}{ PCR verification } \\
\hline & Inner R & TTGACGCGACGACAGATT & & \\
\hline \multirow{3}{*}{ swnR } & UF & CCGCTCGAGCTTGCCCGTTGTTGGTCT & Xho I & \multirow{3}{*}{ Gene deletion } \\
\hline & UR & GGAATTCGTATCGGCTGGCTTAGTG & Eco RI & \\
\hline & DF & GCTCTAGAAAGTTGCGGCTGAAAGAC & Xba I & \\
\hline
\end{tabular}




\begin{tabular}{|c|c|c|c|c|}
\hline & DR & CGAGCTCGACGAGGACGATGGGTAG & Sac I & \\
\hline & TF & GTCAGCCAGGACTTCGGTAT & & \multirow{2}{*}{ PCR verification } \\
\hline & TR & CTGCGTCTCCAGCATCGT & & \\
\hline & Sc-ExF & CGGGATCCATGCGTGTGGCAATTGCTGG & Bam HI & \multirow{2}{*}{ For yeast expression } \\
\hline & Sc-ExR & CGGGATCCCTACAGTATCGAGTCCGGAT & Bam HI & \\
\hline \multirow{6}{*}{ swnNR } & UF & AACTGCAGGTCTTTAATGTGTCGATTCT & Pst I & \multirow{4}{*}{ Double deletion } \\
\hline & UR & CGGGATCCGCGTCTCCAGCATCGTGAA & Bam HI & \\
\hline & DF & GCTCTAGACTACCCATCGTCCTCGTCTC & \multirow[t]{2}{*}{ Xba I } & \\
\hline & DR & GCTCTAGATCGACGTCGCGAAACATTTC & & \\
\hline & Inner F & AGCATGGCATGGTTACTT & & \multirow{2}{*}{ PCR verification } \\
\hline & Inner R & TGCAATGGTGCAAGGTTT & & \\
\hline \multirow{12}{*}{ swnK } & UF & GGAATTCGTCTGGCTGGAAGGGAAAC & Eco RI & \multirow{4}{*}{ Gene deletion } \\
\hline & UR & AACTGCAGACACCACCCGGTAATCGA & Pst I & \\
\hline & DF & GACTAGTTGGTAGTGATGGCTGGAGAC & Spe I & \\
\hline & DR & GACTAGTGCTGAAAGGCATAAGTGGG & Spe I & \\
\hline & $\mathrm{TF}$ & CCATGATACAGCCTACTTCTGA & & $\mathrm{PCP}$ wrification \\
\hline & TR & GAAACTTCCAGGGCAAACA & & PCK vermication \\
\hline & EXF1 & AACAACTAGTTCTAGAATGAGTGGCATCTCAGATCA & & Fusion PCR for \\
\hline & EXR1 & TTCTCGCTTCCCGATTCGGCCCACATGATG & & heterogeneous \\
\hline & EXF2 & CATCATGTGGGCCGAATCGGGAAGCGAGAA & & expression in Beauveria \\
\hline & EXR2 & CCGAGTCAACAACTCCGGCAGCGTGAACTA & & bassiana \\
\hline & EXF3 & TAGTTCACGCTGCCGGAGTTGTTGACTCGG & & \\
\hline & EXR3 & TGGCGGCCGCTCTAGATCACGTACGCATTTTGGCCC & & \\
\hline & UF & CCGCTCGAGGTTTCACTTACAACCGTCTA & Xho I & \\
\hline & UR & GGAATTC TACAATATGTTCAGCCTCAT & Eco RI & Gene deletion \\
\hline $\mathrm{swnH2}$ & DF & GACTAGT GGCAATGGAGGACAAGTA & Spe I & 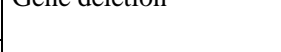 \\
\hline & DR & CGAGCTCCAACGAGTTCAGGGATAAA & Sac I & \\
\hline & Inner F & CATTCTCCACCATTCCCAACC & & PCR verification \\
\hline & Inner R & TGCCGATGACGACTCCTACAAC & & \\
\hline
\end{tabular}




\section{References}

(1) Xia, Y. L., Luo, F. F., Shang, Y. F., Chen, P. L., Lu, Y. Z., and Wang, C. S. (2017) Fungal cordycepin biosynthesis is coupled with the production of the safeguard molecule pentostatin. Cell Chem Biol 24, 1479-1489.

(2) Fang, W., Pei, Y., and Bidochka, M. J. (2006) Transformation of Metarhizium anisopliae mediated by Agrobacterium tumefaciens. Can. J. Microbiol. 52, 623-626.

(3) Huang, W., Shang, Y., Chen, P., Cen, K., and Wang, C. (2015) Basic leucine zipper (bZIP) domain transcription factor MBZ1 regulates cell wall integrity, spore adherence, and virulence in Metarhizium robertsii. J. Biol. Chem. 290, 8218-8231.

(4) Cen, K., Li, B., Lu, Y. Z., Zhang, S. W., and Wang, C. S. (2017) Divergent LysM effectors contribute to the virulence of Beauveria bassiana by evasion of insect immune defenses. PLoS Pathog. 13, e1006604.

(5) Willcott, M. R. (2009) MestRe Nova. J. Am. Chem. Soc. 131, 13180-13180.

(6) Frisch, M., Trucks, G., Schlegel, H., Scuseria, G., Robb, M., Cheeseman, J., Scalmani, G., Barone, V., Mennucci, B., and Petersson, G. (2009) Gaussian 09, Revision D.01. Gaussian, Inc., Wallingford $C T$.

(7) Lodewyk, M. W., Siebert, M. R., and Tantillo, D. J. (2012) Computational prediction of ${ }^{1} \mathrm{H}$ and ${ }^{13} \mathrm{C}$ chemical shifts: a useful tool for natural product, mechanistic, and synthetic organic chemistry. Chem Rev 112, 1839-1862.

(8) Javot, H., Penmetsa, R. V., Terzaghi, N., Cook, D. R., and Harrison, M. J. (2007) A Medicago truncatula phosphate transporter indispensable for the arbuscular mycorrhizal symbiosis. Proc. Natl. Acad. Sci. USA 104, 1720-1725. 